\title{
Tuberculous pericarditis: three cases and brief review
}

\author{
E.I. Metaxas ${ }^{1}$, K. Kotsifas 1 , G. Tatsis 1 , P. Simpsiris², \\ I. Ntanos ${ }^{2}, \mathrm{G}$. Tsoukalas ${ }^{1}$
}

ABSTRACT: Tuberculous pericarditis: three cases and brief review. E.I. Metaxas, K. Kotsifas, G. Tatsis, P. Simpsiris, I. Ntanos, G. Tsoukalas.

Tuberculous pericarditis is a form of extrapulmonary tuberculosis that is considered unusual in western countries. Diagnosis is often challenging, while proper treatment has a major impact on prognosis. We present three interesting cases, with complicated or unusual manifestations of tuberculous pericarditis: a patient with cardiac tamponade as the initial manifestation of tuberculosis; a patient progressing to constriction despite adequate treatment; and a patient receiving anti-TNF $\alpha$, who developed pericarditis in the context of immune reconstitution inflammatory syndrome. We briefly review the clinical features of tuberculous pericarditis, whilst our main focus is on the available diagnostic techniques and treatment aspects. In conclusion, tuberculous pericarditis remains an active thread in the western world. A high index of suspicion combined with the use of all available diagnostic techniques are important to increase diagnostic yield. Prompt and effective treatment is essential to reduce morbidity and mortality. Monaldi Arch Chest Dis 2010; 73: 1, 44-50.

1 Evaggelismos General Hospital, Athens,

2 Sotiria General Hospital of Chest Diseases, Athens, Greece.

Correspondence: Eugenios I. Metaxas, MD; Pulmonary Medicine Department, Evaggelismos General Hospital, Ipsilantou 45-47, Athens, Greece; e-mail: eumetaxas@yahoo.gr

\section{Introduction}

Tuberculous Pericarditis (TP) in western countries, represents up to $4 \%$ of total pericarditis cases [1]. Although considered unusual [2] this percentage tends to increase, mainly as a result of immigration [3]. Its frequency is related to the disease's prevalence in different countries, as well as to the presence of immunosuppression, mainly due human immunodeficiency virus (HIV) infection. In this article we present 3 cases of TP where the patients were recently hospitalised in two hospitals in Athens, Greece. A summary of the characteristics of the 3 patients is presented in table 1. A brief review of diagnostic techniques and therapeutic procedures of TP is also presented.

\section{Cases}

\section{Case 1}

A 25 year-old man from Poland, who had been living in Greece for the past 12 months, presented at the emergency department (ED) complaining of dyspnea, palpitations, thoracic pain, abdominal distention, productive cough of purulent sputum, fever and malaise, for the last 20 days. He had no significant medical history, apart from significant alcohol and tobacco consumption. On physical examination, his arterial blood pressure was 110/80 $\mathrm{mmHg}$, his pulse rate was $170 / \mathrm{min}$, respiratory rate 40 breaths/min and $\mathrm{SpO}_{2}$ of $89 \%$ on room air. He had absent breath sounds on the left hemithorax with dullness to percussion, as well as signs of ascites and significant edema of both lower limbs. The electrocardiogram revealed atrial fibrillation with rapid ventricular response and the chest radiograph (CXR) complete opacification of the left hemithorax and increased cardiothoracic index. No signs of pulmonary tuberculosis were apparent, even after drainage of the pleural space. An echocardiogram revealed the presence of cardiac tamponade and the patient was transferred for surgical treatment. A pericardial window was created and 1.2 litres of pericardial and 4 litres of pleural fluid were drained. Anti-tuberculous chemotherapy with isoniazid (INH), rifampicin (RIF), pyrazinamide (PZA) and streptomycin (STR), together with corticosteroids, was initiated because of a high clinical suspicion of tuberculosis. Pericardial biopsy showed non-necrotic granulomas, while the smear for acid-fast bacilli (AFB) was negative. However, culture of pericardial tissue and fluid were positive for Mycobacterium Tuberculosis which proved sensitive to all primary antituberculous drugs. Bronchoscopic examination was without abnormal findings. Cultures of pleural fluid, bronchial secretions and a gastric aspirate were negative and the tuberculin skin test (TST) was 0 $\mathrm{mm}$. The patient recovered promptly and was released in good clinical condition 20 days after surgery. Unfortunately, he was lost to follow up so the final outcome is unknown. 


\begin{tabular}{|c|c|c|c|}
\hline & Case 1 & Case 2 & Case 3 \\
\hline Predisposing Factors & Alcohol & No & Methotrexate \& Infliximab \\
\hline Presentation & Tamponade & Tamponade & IRIS \\
\hline Tuberculin skin test & $0 \mathrm{~mm}$ & $15 \mathrm{~mm}$ & $12 \mathrm{~mm}$ \\
\hline Treatment & $\begin{array}{l}\text { Standard Treatment } \\
\text { Pericardial Window }\end{array}$ & $\begin{array}{l}\text { Second Line Drugs } \\
\text { Decortication }\end{array}$ & Standard Treatment \\
\hline Adjuvant Steroids & Yes & Yes & Yes \\
\hline Outcome & $\begin{array}{l}\text { Discharged } \\
\text { Lost to follow up }\end{array}$ & Improvement & Cure \\
\hline
\end{tabular}

IRIS: Immune Reconstitution Inflammatory Syndrome.

\section{Case 2}

A 47 year-old man, from Greece, presented at the ED because of dyspnea, fever and ankle edema. He was an ex-smoker and had a history of pulmonary tuberculosis in 1990, when he received a complete course of antituberculous chemotherapy. Clinical examination was compatible with bilateral pleural effusions, while heart sounds were diminished, and there was tachycardia, low arterial blood pressure, distended neck veins and symmetrical lower limb edema. A CXR showed increased cardiothoracic index, bilateral pleural effusions and subtle markings compatible with remote pulmonary TB on both apices. A chest computed tomography (CT) scan confirmed the aforementioned findings, as well as the presence of a large pericardial effusion and enlarged mediastinal lymph nodes. Cardiac ultrasound confirmed the clinical suspicion of tamponade and prompted the performance of a surgical pericardial window. Pericardial biopsy showed granulomas with caseous necrosis and cultures of pericardial tissue and fluid were positive for M. tuberculosis with sensitivity to all primary antituberculous drugs. A TST was $15 \mathrm{~mm}$. Antituberculous chemotherapy with INH, RIF, PZA and EMB, together with steroids was initiated. Two months later EMB and PZA were discontinued and treatment continued with INH and RIF.

However, two months later, the patient was readmitted with right heart failure. A new CT scan revealed a significant pericardial effusion, while an echocardiogram made the diagnosis of effusiveconstrictive pericarditis. The patient reported adherence to treatment and no alcohol nor any other drug or supplement consumption. The patient underwent a technically demanding partial pericardiectomy. Pericardium was thick, up to $1.5 \mathrm{~cm}$, partially calcified. Histology of the pericardium showed the persistence of granulomas and an amplified mycobacterium tuberculosis direct test (AMTD) detected RNA of M. tuberculosis, while smear was negative as were cultures two months later. Pleural fluid was a lymphocytic exudate with adenosine deaminase (ADA) levels $23 \mathrm{U} / \mathrm{L}$. Fever appeared postoperatively with no localising signs. In view of the persistence of pericardial effusion with progression to constriction, active granulomatous inflammation and the presence of mycobacteria in the pericardium, as highlighted by nucleic acid amplification (NAA), treatment failure was considered possible. Second line drugs - Moxifloxacin, Ethionamide, Capreomycin and Cycloserine - were added to INH and RIF. The patient tolerated treatment well and experienced a gradual clinical and hemodynamic improvement. No steroids were then added to treatment; indomethacin was used instead for two months. The injectable agent was discontinued 6 months later and treatment continued with 5 drugs aiming to complete 24 months. The patient remains actually in good clinical condition with only mild functional compromise.

\section{Case 3}

A 49 year-old man from Greece presented with dry cough, low grade fever and malaise. He was a police officer in the immigration department and had daily contact with immigrants. He was an active smoker (25 pack/years) and had a history of psoriatic arthritis for which he was on chronic treatment with methotrexate. For one year, infliximab (anti-TNF $\alpha$ ) every 2 months was added, due to inadequate control. A CXR followed by a chest CT scan revealed a diffuse micronodular pattern together with mediastinal lymphadenopathy and hepatosplenomegaly (figures 1,2 ). A TST, which before the onset of infliximab treatment was non reactive, was measured at $12 \mathrm{~mm}$ and tuberculosis was suspected. Sputum and bronchial washings were negative on direct examination (while positive on culture some weeks later, without drug resistance). Transbronchial biopsies were not per- 


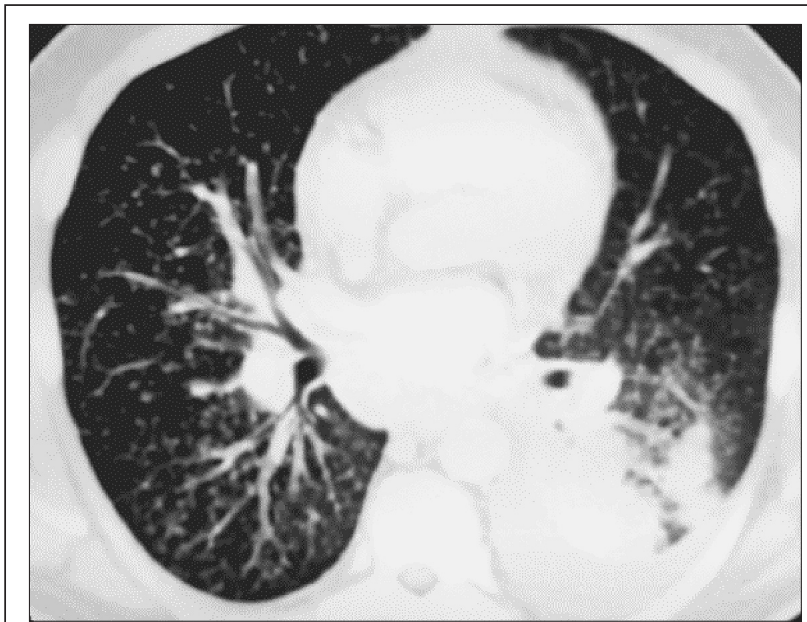

Fig. 1. - Miliary tuberculosis, in a 49 year-old man receiving Infliximab. Also noted are pericardial and left pleural effusions and sizable subcarinal adenopathy.

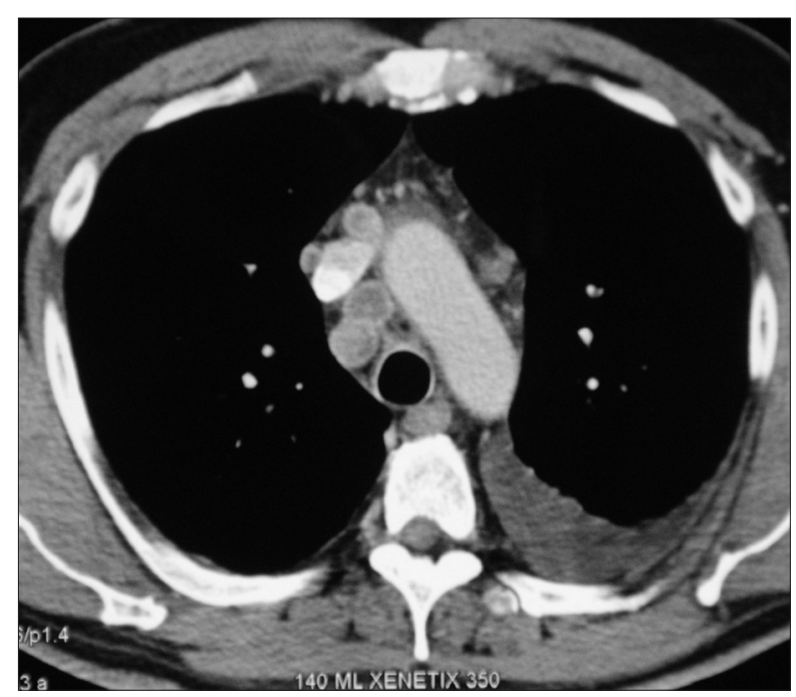

Fig. 2. - Mediastinal lymph nodes with characteristic rim enhancement and hypodense centre on the same patient.

formed. Node sampling through mediastinoscopy revealed granulomatous inflammation with caseous necrosis, with positive Ziehl-Neelsen stain and negative AMTD. The patient was placed on standard anti-tuberculous chemotherapy with INH, RIF, PZA and STR. Treatment with infliximab was discontinued. On follow up two months later, and despite clinical improvement, necrosis of mediastinal lymph nodes and moderate pericardial and left pleural effusions were noted. Echocardiography confirmed the presence of a moderate pericardial effusion without hemodynamic compromise. Sputum was again negative on smear, while culture remained positive, though with reduced colonies. A pleural tap revealed a lymphocytic exudate with ADA levels of 29 U/L. Smear and culture were negative for TB. Antinuclear antibodies were negative.

In view of the clinical improvement, the sensitivity of the strain with all primary drugs and the compatible delay between the onset of antituberculous treatment and the radiological deterioration, Immune Reconstitution Inflammatory
Syndrome (IRIS) was suspected and corticosteroids were added to treatment. INH and RIF continued for an additional 7 months. The patient had an excellent clinical course and the effusions resolved.

\section{Discussion}

TP is a form of extrapulmonary tuberculosis with a mortality of up to $85 \%$ in 6 months if left untreated [4] and with favorable prognosis if appropriate antituberculous chemotherapy is given [5]. It appears to accompany pulmonary tuberculosis in $1-2 \%$ of cases [6]. Occasionally, cardiac tamponade, as seen in cases 1 and 2, is the presenting manifestation of the disease. Up to 2001 , only 80 such cases had been described in the English literature [7]. As the disease progresses, about one half of the patients that did not receive any, or received insufficient treatment, will eventually develop cardiac tamponade [8]. Moreover, tuberculosis is the most frequent cause of restrictive pericarditis (38$83 \%$ of total cases) [9]. Restriction develops in all patients with TP without treatment and in 30-50\% of patients despite antituberculous chemotherapy $[7,10]$. This was probably the case with our second patient. Mycobacteria can gain access to the pericardium in different ways. Most commonly this is through the mediastinal lymph nodes [11]. Mediastinal node inflammation with subsequent central necrosis is a characteristic feature of TP [12] and was present in our second and third cases, where a chest CT was performed. Other routes of infection include hematogenous spread, contiguous spread from the lung, the visceral pleura or a diseased rib [13]. TP develops in four stages: i. Dry, ii. Exudative, iii. Absorptive or early fibrous, and iv. Restrictive or late fibrous [14].

\section{Diagnosis}

Attributing a pericardial effusion to tuberculosis is often challenging. Sensitivity of AFB stain of pericardial fluid varies between $0-42 \%$ [6, 10], whereas specificity is $98 \%$ [15]. Sensitivity of culture from pericardial fluid or pericardial tissue also varies in different publications between 30$100 \%$ [16]. The time of pericardiocentesis is important; the closer to the disease's onset the better are the results [17]. The method and the material of culture are also important [18]. Cultures of both pericardial fluid and tissue add to the sensitivity of each one alone $[19,20]$. Granulomas with caseous necrosis on histopathology, provide definite proof of the disease [21] with sensitivity between 10$87 \%[19,22]$ and $100 \%$ specificity. NAA tests for M. Tuberculosis are an important diagnostic advance. Sensitivity and specificity vary again among studies, between $15-80 \%$ and $75-100 \%$ respectively $[19,23]$. Pericardial tissue offers higher sensitivity than fluid (80\% vs 15\%) [19]. Disadvantages of NAA include possible contamination of the samples and false positive results, and lower sensitivity compared to culture $[23,24]$. 
Indirect diagnostic methods, first applied to pleural effusions, include the measurement of ADA and interferon- $\gamma(\mathrm{INF}-\gamma)$ levels in the pericardial fluid. High ADA levels have been related to TP $[15,22,25]$. Cut-off levels between 30-60 U/L have been used [26]. In a meta-analysis [27] using ADA levels $>40 \mathrm{U} / \mathrm{L}$, sensitivity ranges from $83 \%[23]$ to $100 \%[28,29]$ and specificity from $78 \%[23,28]$ to $100 \%$ [29]. A small study of 12 patients compared the diagnostic value of PCR versus ADA: PCR had smaller sensitivity, $75 \%$ vs $83 \%$, but better specificity, $100 \%$ vs $78 \%$ [23]. Very high ADA levels have been related to increased probability of developing restrictive pericarditis [22]. Their diagnostic value remains high in the context of HIV infection, even if measured levels are lower compared to immunocompetent patients [15].

IFN- $\gamma$ levels in the pericardial fluid provide at least equal results to ADA. A study that set a cutoff level of $50 \mathrm{pg} / \mathrm{L}$, gave sensitivity and specificity of $92 \%$ and $100 \%$ respectively [4]. A second study, using a cut-off level of $200 \mathrm{pg} / \mathrm{L}$, reported an absolute sensitivity and specificity of $100 \%$ [15]. The authors of this study recommended the use of IFN- $\gamma$ as routine and that measurement of ADA should be reserved for cases that IFN- $\gamma$ measurement is not available. However the method is not widely used mainly due to its high cost [26].

The value of TST in the diagnosis of active TB is limited. The results are not reliable in regions where tuberculosis is endemic [24] while in nonendemic regions its usefulness is again questionable [10]. Using a cut-off of $10 \mathrm{~mm}$, sensitivity is $43 \%$ and specificity $93 \%$ [4].

Interferon gamma release assays (IGRAs) have better sensitivity and specificity than TST in the diagnosis of latent tuberculous infection but they still cannot differentiate between latent infection and disease. However, a negative predictive value in the order of $84-87 \%$ can have a useful supplementary role in excluding the diagnosis of TB [30, 31]. Compartmentalisation of sensitised $\mathrm{T}$ cells in the pleural space in cases of pleural tuberculosis, led to investigate the application of IGRAs on effusion, rather than blood, derived monocytes, in an attempt to improve sensitivity and specificity for the diagnosis of pleural TB [32]. Results are slightly improved, but there are still false positive and negative results which compare unfavorably with the more simple and less expensive ADA and IFN- $\gamma$ measurement [33].

\section{Treatment}

Early treatment of TP prevents the development of restrictive pericarditis in about half the patients [14] and increases survival [21]. Because of the serious complications of the disease, the immediate onset of antituberculous chemotherapy is considered necessary. Treatment can be given empirically in the presence of strong clinical suspicion, especially in endemic regions [10]. Treatment includes the use of standard antituberculous treatment, together with corticosteroids [18, 34]. Prolongation of treatment beyond 6 months does not appear to confer advantage [26].

There is evidence for a beneficial role of corticosteroids both in the early effusive and late constrictive stages [34, 35]. However, published trials cannot be considered conclusive and large randomised controlled trials are needed both in HIVpositive and HIV-negative patients [18, 36, 37]. Corticosteroids seem to confer faster recession of symptoms and resorption of the pericardial fluid and reduce the need for invasive procedures to control the pericardial effusion; more importantly, mortality appears significantly reduced [38]. However, it remains doubted whether corticosteroids can inhibit progression to constriction [35, 38]. Concern about the immunosuppressive effect of corticosteroids, together with skepticism on their usefulness lead some authors to argue against their use [8]. If infection from drug resistant mycobacteria is possible, then adequate treatment should be secured before adding corticosteroids [39]. Initial recommended dose is $60 \mathrm{mg}$ of prednisone daily for 4 weeks, followed by $15 \mathrm{mg} /$ day for 2 weeks [20]. Another option is an initial dose of $60 \mathrm{mg}$ of prednisone and progressive tapering during the following 12 weeks [40]. Administration of corticosteroids in patients with HIV infection is also recommended, after careful case-specific assessment and for a shorter duration; again, studies with adequate number of patients do not yet exist $[35,37]$.

\section{Surgical intervention}

Cardiac tamponade is an absolute indication for drainage. Open drainage appears to eliminate the need for repeat pericardiocentesis and may even confer improved outcome, irrespective of the use of steroids [38]. It is a minor procedure and provides tissue specimens that increase the diagnostic yield. Open drainage may therefore be preferable to pericardiocentesis.

The timing of surgical intervention in constrictive TP remains controversial. Based on a significant proportion of patients with favourable course on medical treatment alone, some authors reserve surgery for patients failing after 1 to 2 months of chemotherapy $[10,38]$ On the other hand, long standing constriction enhances the formation of denser and calcified adhesions; furthermore it may alter the underlying myocardium's structure and function. Consequently, some authors advocate early pericardiectomy $[41,42]$. However, perioperative mortality is considerable; it is usually between 6-12\%, but may reach $19 \%$ in the presence of extensive adhesions and calcifications [42, 43]. Advanced age, atrial fibrillation, tricuspid insufficiency, need for inotropic support, low cardiac output, high atrial pressure, ascites, low functional status and the duration of constriction have been related to poor outcomes [42, 43]. Total pericardiectomy is technically more demanding but when feasible has superior results - both early and late compared with partial resection [44]. 


\section{Tuberculosis, anti-TNF and IRIS}

Paradoxical clinical or radiological worsening while on appropriate antituberculous treatment is attributed to the recovery of the patient's immune system. It is a diagnosis of exclusion: treatment failure (especially drug resistance), drug reaction, and a second disease (most commonly another infection), must be ruled out before making a diagnosis of immune reconstitution inflammatory syndrome. IRIS is a well known complication in 28$35 \%$ of HIV positive patients after introducing antiretroviral and antituberculous therapy; it is also observed in about $10 \%$ of HIV negative patients [45]. The median time from onset of anti-tuberculosis treatment to paradoxical reaction is approximately one month for HIV positive and two months for HIV negative patients respectively (range from 2 weeks to 5 months) [45, 46]. Pericarditis in the context of IRIS is uncommon [47]. The association of anti-TNF treatment with tuberculosis is well established and standard antituberculous treatment is indicated [45].

Tuberculosis while on anti-TNF treatment may not only result from reactivation of latent disease but from de novo infection; this might have been the case in our third patient and could explain the conversion of the TST. We therefore suggest periodic surveillance, including a TST and/ or an IGRA assay, in patients with a negative initial screening, commencing anti-TNF treatment.

Cases of IRIS after withdrawing anti-TNF treatment, appeared recently in the literature [48, $49,50]$. Consequently, clinical recommendations on management are not evidence based. As in our case, discontinuation of anti-TNF treatment and prolonged (9-12 months) antituberculous therapy are described; neither is supported by evidence and in fact if tuberculous infection is controlled, pursuing anti-TNF $\alpha$ treatment may be safe while preventing the occurrence of IRIS $[45,48,50]$. Otherwise, systemic corticosteroids may be used, as for HIV positive patients.

In an era of increasing drug resistant tuberculosis, differentiating between treatment failure and IRIS is challenging and of paramount clinical importance [51]. Adding to this challenge, it is suggested that drug resistance and IRIS are not mutually exclusive and may in fact overlap in some patients [39]. Appropriate antituberculous regimen should ideally be secured before adding immunosuppressive treatment for a presumed IRIS [52].

\section{Natural course, treatment failure, or IRIS?}

While in our third patient the diagnosis of IRIS seems certain and the choice of treatment reasonable, in our second patient the therapeutic decisions may be disputed. Constrictive pericarditis was most likely the natural evolution of the disease. The original sensitivity pattern and the inability to grow mycobacteria from pericardium samples at the time of pericardiectomy support this interpretation. On the other hand, the unfavourable course of the disease despite appropriate treatment, including adjunctive steroids and surgical drainage, raised concerns of treatment failure, that were fostered after the detection of mycobacterial RNA on pericardial biopsy after 4 months of treatment. The decision to use second line drugs may have been immoderate; however, the patient did experience a favourable clinical course thereafter, despite an incomplete pericardiectomy and the avoidance of steroid use. IRIS in this patient seems a less likely diagnosis. It would be expected to only cause increase in pericardial effusion rather than constriction; moreover, a four month delay is longer than usually encountered. However, a paradoxical reaction component cannot be absolutely excluded, as IRIS may complicate the course of antituberculous treatment in both immunocompetent and immunocompromised patients, and may even overlap with disease progression. Differential diagnosis between the natural course of TB, treatment failure and IRIS are not always straightforward making therapeutic decisions intricate and vital.

In conclusion, $\mathrm{TP}$ is a very serious disease with considerable morbidity and mortality. It is indeed present in western countries and must always be included in the differential diagnosis of pericarditis. Immigration and immunosuppression are expected to further increase its incidence. Presentation may be acute and impose rapid diagnostic and therapeutic decisions. Physicians must be of knowledge and take advantage of all available diagnostic procedures in order to early diagnose and confront the disease. Restrictive pericarditis may eventually result despite adequate anti-tuberculous treatment; adjuvant steroid treatment may improve outcome. Tuberculous pericardial effusion due to immune reconstitution inflammatory syndrome may be benign but is a diagnosis of exclusion, most importantly of treatment failure.

\section{References}

1. Maisch B. Pericardial diseases, with a focus on etiology, pathogenesis, pathophysiology, new diagnostic imaging methods, and treatment. Curr Opin Cardiol 1994; 9: 379-88.

2. Trautner BW, Darouiche RO. Tuberculous pericarditis: optimal diagnosis and management. Clin Infect Dis 2001; 33: 954-61.

3. Gibbs CR, Watson RD, Singh SP, Lip GY. Management of pericardial effusion by drainage: a survey of 10 years' experience in a city centre general hospital serving a multiracial population. Postgrad Med J 2000; 76: 809-13.

4. Reuter H, Burgess L, Van Vuuren W, Doubell A. Diagnosing tuberculous pericarditis. QJM 2006; 99: 827-39.

5. Long R, Younes M, Patton N, Hershfield E. Tuberculous pericarditis: long-term outcome in patients who received medical therapy alone. Am Heart J 1989; 117: 1133-9.

6. Fowler NO. Tuberculous pericarditis. JAMA 1991; 266: 99-103.

7. Gladych E, Goland S, Attali M, Somin M, Malnick SD. Cardiac tamponade as a manifestation of tuberculosis. South Med J 2001; 94: 525-8. 
8. Yang CC, Lee MH, Liu JW, Leu HS. Diagnosis of tuberculous pericarditis and treatment without corticosteroids at a tertiary teaching hospital in Taiwan: a 14 year experience. J Microbiol Immunol Infect 2005; 38: 47-52.

9. Cinar B, Enç Y, Göksel O, et al. Chronic constrictive tuberculous pericarditis: risk factors and outcome of pericardiectomy. Int J Tuberc Lung Dis 2006; 10: 701-6.

10. Sagrista-Sauleda J, Permanyer-Miralda G, Soler-Soler J. Tuberculous pericarditis: ten-year experience with a prospective protocol for diagnosis and treatment. $J$ Am Coll Cardiol 1988; 11: 724-28.

11. Cherian G, Habashy AG, Uthaman B, Hanna RM. Tuberculous Pericardial Effusion-Mediastinal Lymph Glands: The Cause and Clue to the Etiology. Indian Heart J 2003; 55: 228-33.

12. Cherian G, Uthaman B, Salama A, Habashy AG, Khan NA, Cherian JM. Tuberculous pericardial effusion: features, tamponade, and computed tomography. Angiology 2004; 55: 431-40.

13. Afzal A, Keohane M, Keeley E, Borzak S, Callender $\mathrm{CW}$, Iannuzzi M. Myocarditis and pericarditis with tamponade associated with disseminated tuberculosis. Can J Cardiol 2000; 16: 519-21.

14. Suwan PK, Potjalongsilp S. Predictors of constrictive pericarditis after tuberculous pericarditis. BMJ 1995; 73: $187-89$

15. Burgess LJ, Reuter H, Carstens ME, Taljaard JJ, Doubell AF. The use of adenosine deaminase and interferon-gamma as diagnostic tools for tuberculous pericarditis. Chest 2002; 122: 900-5.

16. Cherian G. Diagnosis of tuberculous aetiology in pericardial effusions. Postgrad Med J 2004; 80: 262-6.

17. Strang G, Latouf S, Commerford P, et al. Bedside culture to confirm tuberculous pericarditis. Lancet 1991; 338: 1600-1.

18. Strang JI. Tuberculous Pericarditis. J Infect 1997; 35: 215-9.

19. Cegielski JP, Devlin BH, Morris AJ, et al. Comparison of PCR, Culture, and Histopathology for Diagnosis of Tuberculous Pericarditis. J Clin Microbiol 1997; 35: 3254-7.

20. Strang JI, Kakaza HH, Gibson DG, et al. Controlled clinical trial of complete open surgical drainage and of prednisolone in treatment of tuberculous pericardial effusion in Transkei. Lancet 1988; 2: 759-64

21. Syed FF, Mayosi BM. A modern approach to tuberculous pericarditis. Prog Cardiovasc Dis 2007; 50: 21836.

22. Komsuoğlu B, Göldelï O, Kulan K, Komsuoğlu SS. The diagnostic and prognostic value of adenosine deaminase in tuberculous pericarditis. Eur Heart J 1995; 16: 1126-30.

23. Lee JH, Lee CW, Lee SG, et al. Comparison of polymerase chain reaction with adenosine deaminase activity in pericardial fluid for the diagnosis of tuberculous pericarditis. Am J Med 2002; 113: 519-21.

24. Ng TT, Strang JI, Wilkins EG. Serodiagnosis of pericardial tuberculosis. QJM 1995; 88: 317-20.

25. Koh KK, Kim EJ, Cho CH, et al. Adenosine deaminase and carcinoembryonic antigen in pericardial effusion diagnosis, especially in suspected tuberculous pericarditis. Circulation 1994; 89: 2728-35.

26. Mayosi BM, Burgess LJ, Doubell AF. Tuberculous Pericarditis. Circulation 2005; 112: 3608-16.

27. Tuon FF, Litvoc MN, Lopes MI. Adenosine deaminase and tuberculous pericarditis - a systematic review with meta-analysis. Acta Trop 2006; 99: 67-74.

28. Dogan R, Demircin M, Sarigül A, Ciliv G, Bozer AY. Diagnostic value of adenosine deaminase activity in pericardial fluids. J Cardiovasc Surg (Torino) 1999; 40: 501-4.
29. Martinez-Vazquez JM, Ribera E, Ocan̆a I, Segura RM, Serrat R, Sagrista J. Adenosine deaminase activity in tuberculous pericarditis. Thorax 1986; 41: 888-9.

30. Lalvani A. Diagnosing tuberculosis infection in the $21 \mathrm{st}$ century: new tools to tackle an old enemy. Chest 2007; 131: 1898-906.

31. Ferrara G, Losi M, D'Amico R, et al. Use in routine clinical practice of two commercial blood tests for diagnosis of infection with Mycobacterium tuberculosis: a prospective study. Lancet 2006; 367: 1328-34.

32. Losi M, Bossink A, Codecasa L, et al. Use of a T-cell interferon-gamma release assay for the diagnosis of tuberculous pleurisy. Eur Respir J 2007; 30: 1173-9.

33. Hooper CE, Lee G, Maskell NA. Interferon-gamma release assays for the diagnosis of TB pleural effusions: hype or real hope? Curr Opin Pulm Med 2009; 15: 358-65.

34. Mayosi BM, Ntsekhe M, Volmink JA, Commerford PJ. Interventions for treating tuberculous pericarditis. Cochrane Database Syst Rev 2002; (4): CD000526.

35. Evans DJ. The use of adjunctive corticosteroids in the treatment of pericardial, pleural and meningeal tuberculosis: Do they improve outcome? Resp Med 2008; 102: 793-800

36. Ntsekhe M, Wiysonge C, Volmink JA, Commerford PJ, Mayosi BM. Adjuvant corticosteroids for tuberculous pericarditis: promising, but not proven. QJM 2003; 96: 593-9.

37. Hakim JG, Ternouth I, Mushangi E, Siziya S, Robertson V, Malin A. Double blind randomised placebo controlled trial of adjunctive prednisolone in the treatment of effusive tuberculous pericarditis in HIV seropositive patients. Heart 2000; 84: 183-8.

38. Strang JI, Nunn AJ, Johnson DA, Casbard A, Gibson DG, Girling DJ. Management of tuberculous constrictive pericarditis and tuberculous pericardial effusion in Transkei: results at 10 years follow-up. QJM 2004; 97 : 525-35.

39. Meintjes G, Rangaka M, Maartens G. Novel relationship between tuberculosis immune reconstitution inflammatory syndrome and antitubercular drug resistance. Clin Infect Dis 2009; 48: 667-76.

40. Dooley DP, Carpenter JL, Rademacher S. Adjunctive corticosteroid therapy for tuberculosis: a critical reappraisal of the literature. Clin Infect Dis 1997; 25: 872-87.

41. McCaughan BC, Schaff HV, Piehler JM, et al. Early and late results of pericardectomy for constrictive pericarditis. J Thorac Cardiovas Surg 1985; 89: 340-50.

42. Çınar B, Enç Y, Göksel O, et al. Chronic constrictive tuberculous pericarditis: risk factors and outcome of pericardiectomy. Int J Tuberc Lung Dis 2006; 10: 701-6.

43. Bozbuga N, Erentug V, Eren E, et al. Pericardiectomy for chronic constrictive tuberculous pericarditis: risks and predictors of survival. Tex Heart Inst J 2003; 30: 180-5.

44. Chowdhury UK, Subramaniam GK, Kumar AS, et al. Pericardiectomy for constrictive pericarditis: a clinical, echocardiographic, and hemodynamic evaluation of two surgical techniques. Ann Thorac Surg 2006; 81: 522-529.

45. Breen RA, Smith CJ, Bettinson H, et al. Paradoxical reactions during tuberculosis treatment in patients with and without HIV co-infection. Thorax 2004; 59: 704-7.

46. Cheng VC, Ho PL, Lee RA, et al. Clinical spectrum of paradoxical deterioration during antituberculosis therapy in non-hiv-infected patients. Eur J Clin Microbiol Infect Dis 2002; 21: 803-9.

47. Rapose A, Sarvat B, Sarria JC. Immune reconstitution inflammatory syndrome presenting as pericarditis and pericardial effusion. Cardiology 2008; 110: 142-144. 
48. Garcia Vidal C, Rodriquez Fernandez S, Martinez Lacasa $\mathrm{J}$, et al. Paradoxical response to antituberculous therapy in infliximab-treated patients with disseminated tuberculosis. Clin Infect Dis 2005; 40: 756-9.

49. Arend S, Leyten E, Franken W. A patient with de novo tuberculosis during anti-tumor necrosis factor-a therapy illustrating diagnostic pitfalls and paradoxical response to treatment. Clin Infect Dis 2007; 45: 1470-5.

50. Belknap R, Reves R, Burman W. Immune reconstitution to Mycobacterium Tuberculosis after discontinuing infliximab. Int J Tuberc Lung Dis 2005; 9: 1057-8.
51. Miller RF, Shahmanesh M, Talbot MD, et al. Progressive symptoms and signs following institution of highly active antiretroviral therapy and subsequent antituberculosis therapy: immune reconstitution syndrome or infection? Sex Transm Infect 2006; 82: 111-6.

52. Swaminathan S, Padmapriyadarsini C, Narendran G, Thomas BE, Anand S, Rajyalakshimi A. Immune reconstitution syndrome following initiation of antiretroviral therapy in a patient with HIV infection and multidrug-resistant tuberculosis. Indian J Chest Allied Sci 2005; 47: 299-304.

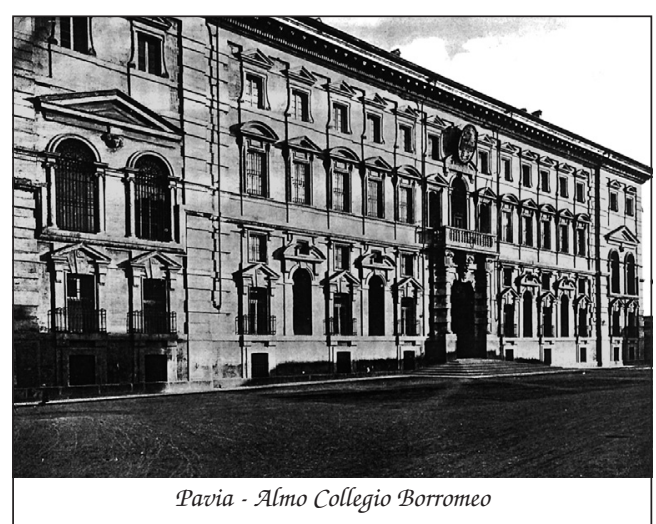

